\title{
Infective endocarditis: some popular tenets debunked?
}

\author{
Susannah J Eykyn
}

Infective endocarditis remains an uncommon disease thus any individual doctor's experience of it must of necessity be limited. Such patients are always "interesting" and invariably stay in hospital for weeks rather than days-this, I think, leads clinicians to assume the infection is more common than it really is. As a (clinical) microbiologist $I$ have been in a unique position to follow all patients with infective endocarditis who have presented or been referred to $\mathrm{St}$ Thomas' Hospital in London since 1970. This has provided a wealth of information as well as the background for this article.

\section{Nomenclature}

The old Oslerian classification of endocarditis as acute, subacute, or chronic was based on the usual course of untreated disease and it is not appropriate today, yet remarkably "SBE" is still used by countless clinicians as a blanket description of patients with endocarditis even when the patient has been ill for only a few days. I know this from perusal of blood culture request forms. The preferred term is infective endocarditis rather than bacterial endocarditis, as fungi at least are outside the latter definition. Native valve infection should be distinguished from prosthetic. Prosthetic endocarditis has conventionally been divided into early and late onset after surgery, with 60 days as the cut off point. This is an unsatisfactory division implying, as it does, that infections acquired at the time of the valve replacement will become manifest within this time whereas it is known that some will not present until much later. A limit of a year has been proposed and this is more realistic. As important as any time limit is the pathogenesis of the infection, whether it has been acquired in the community or as result of a hospital procedure or operation.

\section{Causative pathogens and taxonomic niceties}

Although oral streptococci are the most common pathogens causing native valve endocarditis and community acquired prosthetic valve endocarditis, they are declining in incidence and now account for only about a third of cases of native valve infection. Almost as many cases of native valve infection are caused by staphy-

lococci, most often Staphylococcus aureus, and increasingly coagulase negative strains, ${ }^{1}$ previously regarded by many as pathogens only of prosthetic valves. Remarkably, many papers, even those in the "better" journals, still occasionally refer to "Streptococcus viridans" as though this were a single microbial species. Streptococcal taxonomy is constantly changing and this is of limited interest to clinicians, but oral or viridans streptococcus is a more appropriate description for a member of this group of organisms. Faecal streptococci have been consigned to a new genus, Enterococcus, and thus it is no longer taxonomically correct to refer to Streptococcus faecalis, it is Enterococcus faecalis. Unfortunately taxonomic upheaval has not been limited to streptococci, the coagulase negative staphylococci have also suffered. Older clinicians will remember the (very) old nomenclature of Staphylococcus albus for these microbes. The arrival of convenient (French) kits for speciating staphylococci has meant that virtually any laboratory can now distinguish Staph epidermidis from Staph lugdunensis, Staph simulans, and others. Such speciation is not only of interest to laboratory boffins as it is becoming increasingly clear that certain species of coagulase negative staphylococci (and in particular Staph lugdunensis) can mimic Staph aureus and cause rapidly destructive infection. ${ }^{2}$ A glance at any large series of cases of infective endocarditis will show that although streptococci, enterococci, and staphylococci account for two-thirds of cases, a huge variety of pathogens are possible, particularly in prosthetic valve endocarditis. The St Thomas' series alone can muster cases of Brucella melitensis, Histoplasma capsulatum, Campylobacter fetus, and Erysipelothrix rhusiopathiae. Any microbe can infect a heart valve.

\section{Classical signs (and their absence)}

It is not unusual for the diagnosis of native valve endocarditis to be considered only when the laboratory has reported positive blood cultures (usually of a viridans streptococcus) rather than by clinical suspicion aroused by classical signs. The Oslerian signs of nodes, Janeway lesions, and Roth's spots are now rarely seen in native valve endocarditis and virtually never in prosthetic infection. Non-specific vascular phenomena such as petechiae and splinter haemorrhages are more common but by no means the rule. It is much easier to
Correspondence to:

Dr Eykyn.

Accepted 16 December 1996
Division of Infection Hospital, 
think of the diagnosis of infective endocarditis in a patient who is non-specifically unwell who has a prosthetic valve or a known predisposing heart lesion, than it is in a patient either with an undetected predisposing lesion or none at all. It is also worth emphasising that the presence of viridans streptococci or enterococci in several blood culture bottles from a patient admitted from the community is virtually diagnostic of infective endocarditis.

\section{Dental-induced endocarditis}

It is still generally supposed that most cases of viridans streptococcal endocarditis are caused by dental procedures-this is simply not the case, they result from poor oral hygiene and inflamed gingivae that are susceptible to bleeding. As Wahl ${ }^{3}$ has pointed out, physicians often ask their patients with endocarditis whether they have had any dental treatment in the past six months and if the answer is yes the dental treatment is generally blamed. The period between bacterial invasion and the onset of symptoms in viridans streptococcal endocarditis is relatively short, from a few days to two weeks in the majority of cases. In a review of 200 patients with viridans streptococcal endocarditis, Bayliss et al found only $13 \%$ had received dental treatment within three months of the onset of symptoms and only $8 \%$ within one month, ${ }^{4}$ and Guntheroth found only $4 \%$ had had a dental extraction within two months. ${ }^{5}$ Moreover, many infected patients in these studies had received antibiotic prophylaxis for the dental procedures. It seems increasingly clear that it is poor dental hygiene rather than dental procedures that are responsible for most if not all cases of viridans streptococcal endocarditis.

\section{Antibiotic prophylaxis for endocarditis}

There has never been a placebo controlled study of antibiotic prophylaxis for the prevention of infective endocarditis, and with the ethical and medicolegal difficulties involved, plus the large number of patients required, it is doubtful that one will ever be done, desirable though this might be. The limited retrospective studies of Imperiale and Horwitz, ${ }^{6}$ and of van der Meer $e t a l^{7}$ found a protective efficacy for dental prophylaxis of $91 \%$ and $49 \%$, respectively. There is no information at all on the efficacy of prophylaxis for non-dental procedures. The United Kingdom, in common with many European countries, the United States, and elsewhere, has issued national prophylactic guidelines produced by the Working Party of the British Society for Antimicrobial Chemotherapy (BSAC). The BSAC guidelines are available from the British Heart Foundation (together with a "credit card" style of card for patients about dental prophylaxis), they also feature in the British National Formulary and the Dental Formulary. Not unexpectedly as the various national guidelines have been devised by different groups of "experts" their recommendations are not uniform, but those of the BSAC are among the simplest and most practical. Compliance is said to be improving but is still far from universal. There is also a depressing number of clinicians who institute a "personalised" version of prophylaxis for their patients that is often inappropriate.

\section{How long to treat endocarditis?}

Even though it was shown many years ago in carefully monitored clinical trials that penicillin sensitive viridans streptococcal native valve endocarditis can be satisfactorily treated with a two week regimen of penicillin (oral, intramuscular, or intravenous) plus an aminoglycoside, ${ }^{89}$ this important information seems to have been largely ignored and there is an entrenched belief that six weeks of therapy is needed. Such practice is unacceptable and is inconvenient for patient and hospital alike. Lengthy courses of (usually) very large parenteral doses of penicillin are likely to result in toxicity with fevers, neutropenia, and other ill effects. Failure to respond to antibiotic treatment almost always means that a cardiac surgeon is needed, not an increased dose or change (or both) of antibiotic. Very few cases of infective endocarditis require more than four weeks of antibiotic treatment, and many require less.

\section{Microbiological monitoring of antibiotic treatment}

It is commonly believed in microbiological circles that a full in vitro investigation of the causative microbe (minimum inhibitory and bactericidal concentrations, synergy studies, etc) is mandatory to select an appropriate regimen for all patients with infective endocarditis. For most organisms a routine disc sensitivity is entirely satisfactory and has the merit of being available soon after the organism is isolated from the blood culture. In vitro tests take time and are not routinely done in many laboratories, therefore, they may well give erroneous results, and in most cases contribute little. In practice it is not difficult to select an appropriate regimen for most patients with infective endocarditis. The other microbiological sacred cow is the serum bactericidal titre (back titrations). This has no correlation with clinical cure. For years I used assiduously to perform these tests and "fine tune" the antibiotic therapy accordingly, I have now concluded that it is better to assess the clinical response rather than that of an organism in a test tube.

\section{Culture negative endocarditis}

Cases of infective endocarditis where neither blood culture, excised valve culture, or serology demonstrate a causative pathogen are uncommon, perhaps as few as $5 \%$ of cases. Probably the most common explanation is previous antibiotic treatment that, even if it has not sterilised the valve, will have sterilised the blood cultures. Such cases are most likely to have been caused by viridans streptococci. All patients with infective endocarditis whose blood cultures are sterile should have serological 
investigations for organisms that cannot readily be isolated by conventional culture methods such as Coxiella burnetii, Chlamydia spp and the recently recognised Bartonella (previously Rochalimaea) spp, which incidentally give serological cross reactions with Chlamydia spp. ${ }^{10}$

In conclusion, it is important to emphasise that the greatest advances in the successful management of infective endocarditis since the advent of penicillin have come from cardiology and cardiac surgery. Remarkably, some hospital clinicians are still reluctant to refer patients for specialist care; this is quite unacceptable. As we approach the millennium it would be gratifying if the UK guidelines on prophylaxis and treatment (to which the BSAC working party devote much time) could be followed by more doctors.

1 Etienne J, Eykyn SJ. Increase in native valve endocarditis caused by coagulase-negative staphylococci: an Anglo-
French clinical and microbiological study. Br Heart $\mathcal{f}$ 1990;64:381-4

2 Vandenesch F, Etienne J, Reverdy ME, Eykyn SJ. Endocarditis due to Staphylococcus lugdunensis: report of 11 cases and review. Clin Infect Dis 1993;17:871-6.

3 Wahl MJ. Myths of dental-induced endocarditis. Arch Intern Med 1994;154:137-44.

4 Bayliss R, Clarke C, Oakley C, Somerville W, Whitfield AGW. The teeth and endocarditis. Br Heart $\mathcal{F} 1983 ; 50$ : 506-12.

5 Guntheroth WG. How important are dental procedures as a cause of infective endocarditis? Am $\mathcal{F}$ Cardiol 1984;54: cause of

6 Imperiale TF, Horwitz RI. Does prophylaxis prevent postdental infective endocarditis? A controlled evaluation of dental infective endocarditis? A controlled eval

7 van der Meer JTM, van Wiik W, Thompson J, Vandenbroucke JP, Valkenburg HA, Michel MF. Efficacy of antibiotic prophylaxis for prevention of native-valve endocarditis. Lancet 1992;339:135-9.

8 Tan JS, Terhune CA, Kaplan S, Hamburger M. Successfu two-week treatment schedule for penicillin-susceptible Streptococcus viridans endocarditis. Lancet 1971;ii: 1340-3.

9 Wilson WR, Thompson RL, Wilkowske CJ, Washington II JA, Giuliani ER, Geraci JE. Short-term therapy for streptococcal infective endocarditis. Combined intramuscular administration of penicillin and streptomycin. $f A M A$ 1981;245:360-3.

10 Drancourt $M$, Mainardi $J L$, Brouqui $P$, Vandenesch $F$, Carta A, Lehnert F, et al. Bartonella (Rochalimea) quinCarta A, Lehnert F, et al. Bartonella (Rochalimea) quin1995;332:419-23.

\section{STAMPS IN CARDIOLOGY}

\section{Rudolf Karl Virchow (1821-1902)}

(A)

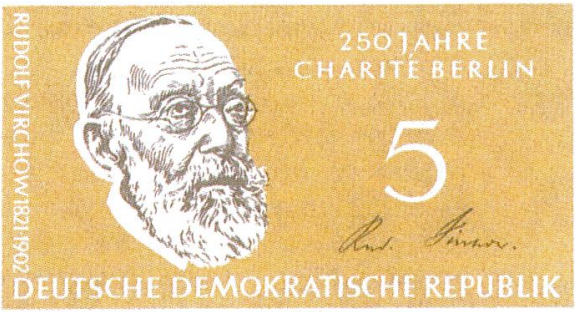

In 1960 East Germany issued a set of five stamps to commemorate the 250th anniversary of Berlin Charity and the 150th Anniversary of Humboldt University in Berlin. The 10 pfennig stamp depicted Robert Koch and the 20 pfennig stamp the University building and statues of William and Alexander von Humboldt. The 5 pfennig value featured Rudolf Virchow (A).

As part of the Celebrities' Birth Anniversaries issue appearing in 1971 East Germany again honoured Virchow with a stamp (B) to mark the 150th anniversary of his birth.

Rudolph Karl Virchow was a great pathologist whose worldwide fame was first established by his concept that cells are the basic unit of life and that a study of cell changes must be the cornerstone of pathology. He pioneered the use of the microscope for this purpose and wrote an acclaimed book Die Cellularpathologie.

Virchow was the first to realise and prove that clots in pulmonary arteries had not been formed there but came from leg and pelvic veins. He noted that the symptoms of pulmonary embolism ranged from sudden death to silent emboli and he related these to the pathological findings. He produced experimental emboli in the dog and later showed that phlebitis was a mechanical and not, as was then thought, an infective process. The terms "embolism" and "thrombosis" were invented by him to describe these processes. Virchow's triad identifies the three main causes of venous thrombosis. He was the first to show that arterial obstruction was often due to emboli, especially with endocarditis. In 1846 he founded the famous journal Archiv fur

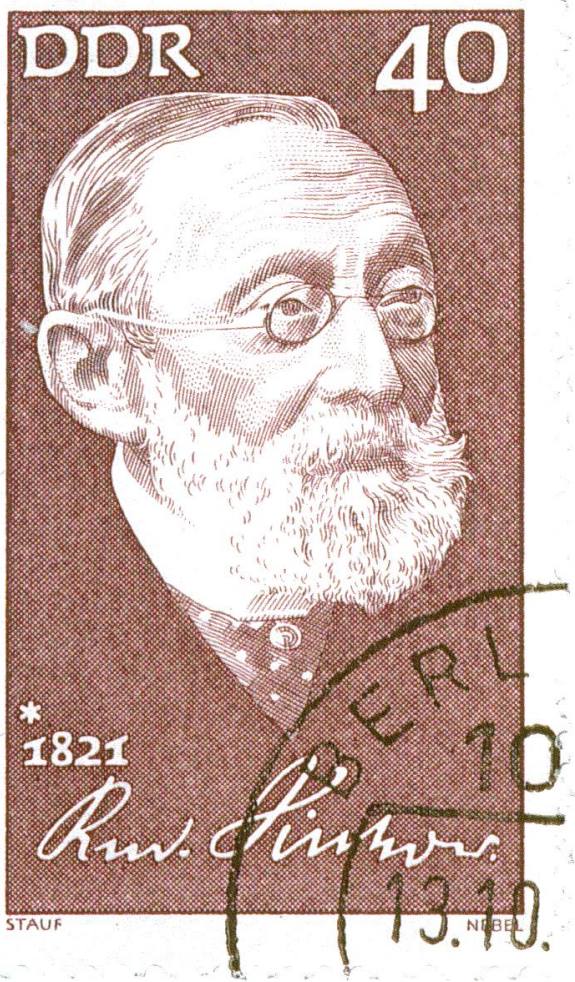

Pathologische Anatomie und Physiologie und fur Klinische Medizin which has been published continuously ever since. It is usually known as Virchow's Archiv and the full title indicates the breadth of his interests. Authors can still benefit from his dictum "Brevity is the best guarantee of being read". He was a politician, serving in the Reichstag for 13 years as leader of the Radical party and was an outspoken opponent of Bismarck. Finally one can note that he pronounced his name "Veer-show", and was unhappy when Lord Lister referred to him in public as "Wir-chov". $M$ K DAVIES A HOLLMAN 